\title{
EFFECT OF PROBIOTIC ON BENEFICIAL AND PATHOGENIC MICROORGANISMS IN THE GASTROINTESTINAL TRACT OF COMMERCIAL BROILER WITH BCRDV VACCINE
}

\author{
M. R. Islam, M. Mostofa*, K. S. Mondal, R. R. Roy, M. G. Sorwar and S. Islam \\ Department of Pharmacology, Bangladesh Agricultural University \\ Mymensingh-2202, Bangladesh
}

\begin{abstract}
The present study was conducted to determine the effect of probiotic on beneficial and pathogenic microorganisms in the gastrointestinal tract of Commercial Broiler. A total of 80 day-old broiler chicks were divided into two main groups I and II with 40 chicks in each. Group I was supplemented with Probiotic (Saccharomyces cerevisiae $2 \mathrm{gm} / \mathrm{kg}$ feed) containing feed and chicks of group II was supplemented with conventional feed. Group I and II was again subdivided into two subgroups as group A, B and C, D respectively on 6 days of age. Birds of subgroups A and C were vaccinated with $N D$ vaccine (BCRDV) and birds of subgroups $C$ and $D$ were kept as unvaccinated. The result evidenced the following information: (a) The total viable count (TVC) and total coliform count (TCC) were significantly $(\mathrm{P}<0.01)$ lower in experimental birds as compared to control at 14, 28 and 32 days of age both in vaccinated and non-vaccinated birds. It was always found that the lower TVC in probiotic fed broilers as compared to conventional fed broilers (b) The total lactobacilli count $(\mathrm{TLC})$ was significantly $(\mathrm{P}<0.01)$ higher in experimental birds as compared to control at 14, 28 and 32 days of age both in vaccinated and nonvaccinated birds. So it can be said that Saccharomyces cerevisiae supplementation can reduce the pathogenic microorganism and increase the growth of beneficial microorganism.
\end{abstract}

Key Words: Probiotic, Broiler, Bacterial count

\section{INTRODUCTION}

Poultry industry has emerged as an agri-business sector started practically during 1980 in Bangladesh. Although poultry industry is expending in Bangladesh, it is facing a lot of constraints. Quality feed is a major problem, which has direct influences on production cost. Feed additives, like antibiotics, hormones and corticosteroids are used in the diet of poultry to improve the performance of poultry production but antibiotics and hormones have harmful effects on poultry as well as human health after consumption. Recently it is believed that probiotic have beneficial effects to improve the productive performance of poultry. A great deal of attention has recently been received from nutritionists and health

* Corresponding author (Email: mostofa57@yahoo.com) 
care experts for proper utilization of nutrients and the use of probiotic for growth promotion of poultry Kabir (2005). The present study is attempted to evaluate the effect of probiotics with regard to clearing bacterial infections and regulating intestinal flora by determining the total viable count (TVC) and total lactobacillus count (TLC) of the crop and cecum samples of probiotics and conventional fed groups Kabir (2005).

\section{MATERIALS AND METHODS}

\section{Experimental birds}

Day-old "Ross 308" broiler chicks were purchased from a market of Gazipur, Bangladesh and transported carefully to the Poultry Shed of the Department of Pharmacology, Bangladesh Agricultural University (BAU), Mymensingh, in well-ventilated paper cartons. The birds were reared under hygienic management practices throughout the entire period of study.

\section{Diets}

Commercially available poultry feed (Quality Feeds Ltd., Dhaka) was used throughout the experimental study. The broiler chicks were fed with standard broiler starter up to 14 days and broiler grower from 15-32 days of age, as formulated by Quality Feeds Ltd., Dhaka.

\section{Probiotic}

Probiotic (Saccharomyces cerevisia) marketed by Wilts Marketing Co., Ltd. and manufactured by Varied Industries Corp., USA was used in this study. A minimum of $1.5 \times 10^{12} \mathrm{CFU} / \mathrm{g}$ (Colony Forming Units) is present in A Max Yeast Culture ${ }^{\mathrm{TM}}$.

\section{Management}

Probiotic was added in feed at a level of $2 \mathrm{gm} / \mathrm{kg}$ and was given to birds of Group I up to 32 days of age. The remaining Group II was kept as control without adding probiotic in feed.

\section{ND vaccine}

Bangla BCRDV was collected from Microbiology laboratory of BAU, Mymensingh, Bangladesh. FnF Pharmaceuticals Limited, Jhenidah, Bangladesh, manufactured Bangla BCRDV. Birds of subgroup A and $C$ were vaccinated with BCRDV through intraocular route.

\section{Body weight of birds}

The live body weight of five birds from each subgroup was taken with the help of standard balance. The body weight of birds was taken at 7 days interval from day 0 to 32 .

\section{Samples for bacterial culture}

Crop and cecum were used as samples for bacterial culture. 


\section{Media and reagents used for bacteriological study Preparation of samples for bacteriological studies}

Portions of crop and cecum with their contents were obtained aseptically with a sterile scalpel and forceps. These portions were homogenized uniformly using a mortar and pestle. From the homogenized mass a one-gram portion was transferred to a sterile tube containing $9 \mathrm{ml}$ of $0.1 \%$ peptone water. Thus 1: 10 dilution of the sample was obtained. Later on serial dilutions of each sample in $0.1 \%$ peptone water were made as per recommendation of International Organization for Standardization (ISO, 1995).

\section{Enumeration of total viable count (TVC)}

For the determination of total bacterial counts, $0.1 \mathrm{ml}$ of each ten-fold dilution was transferred and spread on triplicate PCA or NA agar using a sterile pipette for each dilution. The diluted samples were spreaded as quickly as possible on the surface of the plate with a sterile glass spreader. One sterile spreader was used for each plate. The plates were then kept in an incubator at $35^{\circ} \mathrm{C}$ for $24-48$ hours. Following incubation, plates exhibiting 30-300 colonies were counted. The average number of colonies in a particular dilution was multiplied by the dilution factor to obtain the total viable count. The total viable count was calculated according to ISO (1995). The results of the total bacterial count were expressed as the number of organism of colony forming units per gram (CFU/gm) of crop and cecum samples.

\section{Enumeration of total lactobacillus count (TLC) and total coliform count (TCC)}

For the determination of total lactobacillus count and total coliform count the procedures of sampling, dilution and Streaking was similar to those followed in the total bacterial count. Only in case of lactobacillus count MRS agar and of total coliform count MacConkey agar was used. To perform MPN technique $1 \mathrm{ml}$ of each tenfold dilution was transferred to $9 \mathrm{ml}$ MacConkey broth. For each dilution five test tube containing $9 \mathrm{ml}$ MacConkey broth were used. All the test tubes were incubated at $30^{\circ} \mathrm{C}$ temperature for 48 hours. Growth of the organism was confirmed by the appearance of turbidity. Results were calculated from MPN tables.

\section{Examination of bacterial isolates obtained from samples}

The quantitation of bacteria in crop and cecum was done according to the standard method (ICMSF, 1985). The examination followed detail study of cultural characteristic including colony formation, staining reactions and biochemical properties. In order to find out different types of microorganisms in meat different kinds of bacterial colonies were isolated in pure culture and identified as per instruction of Cowan (1985).

\section{Gram's staining}

Gram's Method of staining was followed during the experiment for the morphological study of bacteria to provide basic information about the presumptive identification as per recommendation of Cowan (1985). 


\section{Carbohydrates fermentation test}

This test is used to differentiate those organisms that oxidize carbohydrates (aerobic utilization) from those organisms that ferment carbohydrates (anaerobic utilization). The test organism is inoculated into two tubes of a tryptone or peptone agar medium containing glucose and the indicator bromothymol blue. One tube is sealed with a layer of liquid paraffin to exclude oxygen. Fermentative organisms utilize the carbohydrates in both the open and sealed tubes whereas oxidative organisms unable to use glucose in sealed tubes. Change of the color of the medium from green to yellow is indicative of positive test.

\section{IMViC test for enteric bacteria}

An amount of $5 \mathrm{ml}$ of MIU medium was inoculated with a smooth colony of the test organism by using a sterile straight wire. Indole paper strip was placed in the neck of MIU tube. The tube is closed with a stopper and incubated at $35-37^{\circ} \mathrm{C}$ overnight. In indole positive case the strip became red, and in urease positive case the medium turned into reddish color. The motile bacteria produced turbidity throughout the medium.

An amount of $2 \mathrm{ml}$ of MRVP broth was taken in test tubes. In two of the test tubes, test organisms were inoculated and these were incubated at $35^{\circ} \mathrm{C}$ for 48 hours. After incubation one of the tubes, 5 drops of methyl red were added and the color was observed. To the other tube $0.6 \mathrm{ml}$ of V-P reagent 1 (creatine) and $0.2 \mathrm{ml} \mathrm{V-P} \mathrm{regent} 2$ ( $40 \% \mathrm{w} / \mathrm{v}$ sodium hydroxide) were added. The tubes were gently shaken with the caps off to expose the medium to oxygen in order to oxidize the aceton. The tubes were allowed to stand for 15-30 min for any color development.

An amount of 3-5 $\mathrm{ml}$ of sterile Koser's citrate medium was inoculated with a broth culture of the test organisms. The inoculated broth culture was incubated at $35-37^{\circ} \mathrm{C}$ for up to 4 days, checking daily for growth. Turbidity and change in color of the indicator showed growth of the organisms in the medium from light green to blue, due to the alkaline reaction following citrate utilization.

\section{TSI agar slant reaction}

After isolation on the selective media, differential-screening media such as triple sugar iron agar was used to further categorize organisms. Colonies were inoculated by stab or streak into triple sugar iron (TSI) agar slants. In TSI agar, if the organisms ferment only glucose the tube will turn yellow in a few hours. The bacteria quickly exhaust the limited supply of glucose and start oxidizing amino acids for energy, giving off ammonia as an end product. Oxidation of amino acids increases the $\mathrm{pH}$ and the indicator in the slanted portion of the tube will turn back to red. The butt will remain yellow. If the organism in the TSI slant ferments lactose and/or sucrose, the butt and the slant will turn yellow and remain yellow for days due to the increased level of acid production. Gas production of the organisms can be ascertained by the appearance of bubbles in the agar. TSI can also be used to indicate whether hydrogen supplied $\left(\mathrm{H}_{2} \mathrm{~S}\right)$ has been produced due to the reduction of sculpture containing compounds. $\mathrm{H}_{2} \mathrm{~S}$ reacts with the ferrous soleplate of the medium producing ferric supplied a black precipitate. 


\section{Motility test}

The motility test was performed to differentiate motile bacteria from non-motile one. Before the test, a pure culture of the organism was allowed to grow in nutrient broth. One to two drops of broth culture was placed in the special slide used for hanging drop method. The slide was examined carefully under 100 power objective of the microscope with immersion oil. The motile organism was identified.

\section{Maintenance of stock culture}

For the maintenance of stock culture, nutrient agar (NA) and MRS agar slants were used. One slant was used for individual isolate and was kept at room temperature. Finally sterile mineral oil was overlaid and the culture was kept at refrigeration temperature until use.

\section{Statistical data analysis}

Data were analyzed using SPSS v.10 for Windows (SPSS Inc., Chicago, IL, USA). Statistically significant differences between group means were determined by analysis of variance (ANOVA). When the differences were significant, Duncan's multiple range tests was performed. Mean values were considered significantly different at $\mathrm{P}<0.05$. Data are expressed as mean values \pm SEM.

\section{RESULTS}

The total viable count (TVC) and total lactobacilli count (TLC) and total coliform count (TCC) in crop and cecum determined. TVC in crop samples were $\log 7.40, \log 7.58, \log 8.14$ and $\log 8.24$ at day $14 ; \log 7.79, \log 7.89, \log 8.20$ and $\log 8.22$ at day $28 ; \log 6.98, \log 6.62$, $\log 8.16$ and $\log 8.14$ at day 32 of age of the birds of subgroups A, B, C and D, respectively. The highest TVC of crop was found in birds of subgroup D at day 28 and the lowest was in crop of birds of subgroup A at day 32 compared to other subgroups. Similarly the TVC in cecum samples were $\log 6.96, \log 7.28, \log 8.24$ and $\log 8.14$ at day $14 ; \log 7.70, \log 7.78, \log$ 8.12 and $\log 8.24$ at day $28 ; \log 6.36, \log 6.74, \log 8.22$ and $\log 8.12$ at day 32 of age of the birds of subgroups A, B, C and D, respectively. The highest TVC of cecun was in birds of subgroup $C$ at day 14 and the lowest was in birds of subgroup $A$ at day 32 compare to other subgroups.

The total lactobacilli count (TLC) in crop samples were $\log 6.98, \log 7.14, \log 3.10$ and $\log 3.4$ at day $14 ; \log 7.56, \log 7.68, \log 3.52$ and $\log 3.54$ at day $28 ; \log 6.98, \log 6.50 \log 3.54$ and $\log 3.44$ at day 32 of age of the birds of subgroups A, B, C and D, respectively. The highest TLC of crop was in birds of subgroups B at day 28 and the lowest TLC were in birds of subgroup $C$ at day 14 compared to other subgroups. Similarly the TLC in cecum samples were $\log 6.72, \log 6.98, \log 3.14$ and $\log 3.26$ at day $14 ; \log 7.51, \log 7.63, \log 3.43$ and $\log 6.62$ at day $28 ; \log 6.32, \log 6.68, \log 3.44$ and $\log 3.42$ at day 32 of age of the birds of subgroups A, B, C and D, respectively. The highest TLC of cecun was in birds of subgroup B at day 14 and the lowest was in birds of subgroup $C$ at day 32 compared to other subgroups. 
The total coliform count (TCC) in crop samples were $\log 3.48, \log 3.24, \log 5.28$ and $\log 5.62$ at day $14 ; \log 3.36, \log 3.62, \log 5.56$ and $\log 5.42$ at day $28 ; \log 3.48, \log 3.42 \log 532$ and $\log$ 5.48 at day 32 of age of the birds of subgroups A, B, C and D, respectively. The highest TCC of crop was in birds of subgroup $C$ at day 28 and the lowest was in birds of subgroup B of at day 14 compared to other subgroups. Similarly the TCC in cecum samples were $\log 3.34$, $\log 3.18, \log 5.12$ and $\log 5.42$ at day $14 ; \log 3.32, \log 3.18, \log 5.28$ and $\log 5.24$ at day $28 ; \log$ 3.44, $\log 3.36, \log 5.18$ and $\log 5.28$ at day 32 of age of the birds of Subgroups A, B, C and D, respectively. The highest TCC of cecun was in birds of subgroup D at day 28 and 32 and the lowest was in birds of subgroup B at day 14 and 28 compare to other subgroups.

\section{Frequency distribution of isolated bacteria in crop and cecum samples}

A total of 94 bacteria were isolated from the crop and cecum samples of bird fed with Probiotics containing feed (Table). The isolated bacteria from crop and cecum were Lactobacilli (82.98\%), Streptococci (4.25\%), Staphylococci $(3.51 \%)$ and Escherichia coli (3.19\%). About 202 bacteria were isolated from the crop and cecum samples of the birds fed with conventional feed (Table 1) during entire period of study from 0 to 32 days of age and were Lactobacilli (19.30\%), Staphylococci (17.32)\%; Streptococci (13.86), Bacilli (12.87\%), Escherichia coli $(25.74 \%)$, Micrococci $(3.46 \%)$, Salmonella $(2.97 \%)$, Proteus $(2.47 \%)$ and unidentified (1.98\%).

Table 1. Frequency distribution of bacterial flora isolated from crop and cecum

\begin{tabular}{|c|c|c|c|c|c|}
\hline \multirow[t]{2}{*}{ Sl. No. } & \multirow[t]{2}{*}{ Name of isolates } & \multicolumn{2}{|c|}{ Number of isolates } & \multicolumn{2}{|c|}{ Percentages (\%) } \\
\hline & & PFG $(A+B)$ & $\mathrm{CFG}(\mathrm{C}+\mathrm{D})$ & PFG $(A+B)$ & $\mathrm{CFG}(\mathrm{C}+\mathrm{D})$ \\
\hline 1. & Lactobacillus spp. & 78 & 39 & 82.98 & 19.30 \\
\hline 2. & Staphylococcus spp. & 5 & 35 & 5.31 & 17.32 \\
\hline 3. & Streptococcus spp. & 4 & 28 & 4.25 & 13.86 \\
\hline 4. & Bacillus spp. & 4 & 26 & 4.25 & 12.87 \\
\hline 5. & Escherichia coli. & 3 & 52 & 3.19 & 25.74 \\
\hline 6. & Micrococcus spp. & 0 & 7 & 0.00 & 3.46 \\
\hline 7. & Salmonella spp. & 0 & 6 & 0.00 & 2.97 \\
\hline 8. & Proteus spp. & 0 & 5 & 0.00 & 2.47 \\
\hline 9. & Others (Unidentified) & 0 & 4 & 0.00 & 1.98 \\
\hline & Total & 94 & 202 & 100 & 100 \\
\hline
\end{tabular}

PFG: Probiotics fed group, A: Vaccinated birds, B: Unvaccinated birds, CFG: Conventional fed group, C: Vaccinated birds and D: Unvaccinated bird

\section{DISCUSSION}

The highest TVC of crop was found in birds of subgroup D at day 28 and the lowest was in crop of birds of subgroup A at day 32 compared to other subgroups. The highest TVC of cecum was in birds of subgroup C at day 14 and the lowest was in birds of subgroup A at day 32 compare to other subgroups. The TVC were significantly lower in experimental birds 
as compared to control at 14,28 and 32 days of age both in vaccinated and non-vaccinated birds. It was always found that the lower TVC in probiotics fed broilers as compared to conventional fed broilers. The present finding of the present study support the finding of Mahajan et al.(2000); Demicho et al. (2005) and Zhang et al. (2005) who found lower microbial count with introduction of probiotics in feed.

The highest TLC of crop was in birds of subgroups B at day 28 and the lowest TLC were in birds of subgroup $C$ at day 14 compared to other subgroups. The highest TLC of cecun was in birds of subgroup B at day 14 and the lowest was in birds of subgroup C at day 32 compared to other subgroups. The TLC was significantly higher in experimental birds as compared to control at 14, 28 and 32 days of age both in vaccinated and non-vaccinated birds. It was always found that the higher TLC in probiotics fed broilers as compared to conventional fed broilers. Kabir (2005) demonstrated the probiotics reduce the total counts of bacteria and E. coli and increase Lactobacilli concentrations in the caecal digesta and also stated that Saccharomyces cerevisiae directly influence the growth of beneficial microorganism like Lactobacillus spp. and lower the growth of E. coli. The present finding of the study support the finding of Juven et al. (1991); Mahajan et al. (1999) and Zhang et al. (2005) who found lower microbial count with introduction of probiotics in feed.

The highest TCC of crop was in birds of subgroup C at day 28 and the lowest was in birds of subgroup B of at day 14 compared to other subgroups The highest TCC of cecum was in birds of subgroup D at day 28 and 32 and the lowest was in birds of subgroup B at day 14 and 28 compare to other subgroups. The TCC were significantly lower in experimental birds as compared to control at 14, 28 and 32 days of age both in vaccinated and non-vaccinated birds. It was always found that the lower TCC in probiotics fed broilers as compared to conventional fed broilers. Canalli et al. (1996) stated that faecal coliforms decreased significantly in the intestine of chickens supplied with probiotics containing feed compared to control. Similar findings were also demonstrated by Mahajan et al. (2000); Santin et al. (2001) and Vicente et al. (2007).

Percentage distribution of selected bacteria was obtained from crop and cecum samples of probiotics and conventional fed broilers. The percentage distribution of selected bacteria found in crop and cecum samples of conventional fed broilers are Lactobacilli $19.30 \%$, Staphylococci $17.32 \%$, Streptococci $13.86 \%$, Bacilli $12.87 \%$, Escherichia coli $25.74 \%$, Micrococci $3.46 \%$, Salmonella 2.97\%, Proteus $2.47 \%$ and Unidentified 98\%. and Lactobacilli $82.98 \%$, Staphylococci 5.31\%, Streptococci 4.25\%; Bacilli 4.25\%, Escherichia coli, 3.19\% in the samples of probiotics containing feed groups. It is noted that Micrococcus, Salmonella, Proteus and unidentified were not found in crop and cecum samples of probiotics fed broilers. In these studies Lactobacilli showed the highest percentage of occurrence. The count of coliforms and enterococci decreased during feeding of the acidophilus milk was reported by Patidar and Prajapati (1999). On the other hand the presence of high percentage of pathogenic Escherichia coli, Staphylococci, Streptococci and Bacilli in addition to the presence of Micrococci, Salmonella and Proteus are alarming for poultry industry. Many researchers are also of the same view that the presence of the above organisms could be potential hazard not only to human health but also to establishment of poultry industry. 
The present research work was concluded that Probiotic increases the beneficial microorganisms and decreases the pathogenic microorganisms in the gastrointestinal tract of commercial broiler.

\section{REFERENCES}

Audisio, C. M., Oliver, G. and Apella, C. M. 2000. Protective effect of Enterococcus faecium J-96, a potential probiotics strain, on chicks infected with Salmonella pullorum. Journal of Food Protection, 63(10): 1333-1337.

Cowan, L. S., Flemming, J. S., Mira, R. T. and Basile, L. F. 1985. Changes in the intestinal Microflora of broiler chickens given a probiotics in feeds. Revista do Sector de Ciencias Agrarias, 15: 125-132.

Cross, M. L. 2002. Microbes versus microbes: immune signals generated by probiotics lactobacilli and their role in protection against microbial pathogens. FEMS Immunology and Medical Microbiology, 34(4): 245-253.

Endo, T. and Nakano, M. 1999. Influence of a probiotics on productivity, meat components, lipid metabolism, caecal flora and metabolites, and raising environment in broiler production. Animal Science Journal, 70(4): 207-218.

Francis, C., Janky, D. M., Arafa, A. S. and Harms, R. H. Interrelationship of LACTOBACILLUS and zinc bacitracin in diets of turkey poults. Poult. Sci., 1978; 57: 1687-1689.

Fuller, R. The importance of lactobacilli in maintaining normal microbial balance in the crop. Br. Poult. Sci., 1977; 18: 85-94.

Hamilton, J. M. T., Shan, S. and Winkler, J. T. 1999. Public health issues arising from microbiological and labeling quality of foods and supplements containing probiotics microorganisms. Public Health Nutrition, 2(2): 223-229.

J. Vicente, J. P., Higgins, S. E., Vicente, J. L., Wolfenden, A. D., Tellez, G. and Hargis, B. M. Temporal effects of lactic acid bacteria probiotic culture on SALMONELLA in neonatal broilers. Poult. Sci. 2007; 86: 1662-1666.

Santin, A., Vahjen, W. and Simon, O. 2001. Growth behaviour of a spore farming probiotics strain in the gastrointestinal tract of broiler chicken and piglets. Archives of Animal Nutrition, 54(1): 1-17.

Juven, B. J., Meinersmann, R. J. and Stern, N. J. 1991. Antagonistic effects of Lactobacilli and Pediococci to control intestinal colonization by human entero-pathogens in live poultry. Journal of Applied Bacteriology, 70(2): 95-103.

Kizerwetter-Swida, M. and Binek, M. Protective effect of potentially probiotic LACTOBACILLUS strain on infection with pathogenic bacteria in chickens. Pol. J. Vet. Sci., 2009; 12: 15-20.

Cowan, Y. S., Kim, Y. R., Ahn, B. K., Kim, M. S. and Kang, C. W. 2000. Effect of dietary supplementation of probiotics [MS102(R)] on performance, blood cholesterol level, size of small intestine and intestinal microflora in broiler chicks. Korean Journal of Animal Science, 42(6): 849-858.

Liangping, S., Lunjiang, Z., Guoping, L., Fanping, L., Shao, L. P., Zhou, L. J., Li, G. P. and Lin, F. P. 2000. Effect of dietary mannan-oligosaccharide and Enterococcus faecium on cell mediated immunity, intestinal microflora and $\mathrm{p}^{\mathrm{H}}$ in chickens. Chinese Journal of Veterinary Science, 20(1): $58-61$. 
Mahajan, P., Sahoo, J. and Panda, P. C. 1999. Effect of probiotic (Lacto-Sacc) feeding, packaging methods and season on the microbial and organoleptic qualities of chicken meat balls during refrigerated storage. Journal of Food Science and Technology Mysore, 37(1): 67-71.

Mahajan, P., Sahoo, J. and Panda, P. C. 2000. Effect of probiotics (Lacto-Sacc) feeding and seasons on poultry meat quality. Indian Journal of Poultry Science, 35(3): 297-301.

Mountzouris, K. C., Tsirtsikos, P., Kalamara, E., Nitsch, S., Schatzmayr, G. and Fegeros, K. Evaluation of the efficacy of probiotic containing LACTOBACILLUS, BIFIDOBACTERIUM, ENTEROCOCCUS, and PEDIOCOCCUS strains in promoting broiler performance and modulating cecal microflora composition and metabolic activities. Poult. Sci., 2007; 86: 309-317.

Panda, A. K., Reddy, M. R., Rao, S. V. R., Raju, M. V. L. N. and Praharaj, N. K. 2000. Growth, carcass characteristics, immunocompetence and response to Escherichia coli of broilers fed diets with various levels of probiotics. Archiv-fur-Geflugelkunde, 64(4): 152-156.

Patidar, S. K. and Prajapati, J. B. 1999. Effect of feeding lactobacilli on serum antibody titer and faecal microflora in chicks. Microbiologie, Aliments, Nutrition, 17(2): 145-154.

Samanya, M. and Yamauchi, K. Histological alterations of intestinal villi in chickens fed dried BACILLUS SUBTILIS var. NATTO. Comp. Biochem. Physiol. Physiol., 2002; 133: 95-104.

Shao, L. P., Zhou, L. J., Li, G. P. and Lin, F. P. 2000. Effect of dietary mannan-oligosaccharide and Enterococcus faecium on cell mediated immunity, intestinal microflora and $\mathrm{pH}$ in chickens. Chinese Journal of Veterinary Science, 20(1): 58-61.

Soerjadi, A. S., Stehman, S. M., Snoeyenbos, G. H., Weinack, O. M. and Smyser, C. F. 1981. The influence of lactobacilli on competitive exclution of paratyphoid salmonellae in chickens. Avian Disease, 25: 1227-1033.

Tellez. G. 2007. Effect of a lactobacillus spp-based probiotic culture product on broiler chicks performance under commercial conditions. International Journal of Poultry Science, 6(3): 154-156.

Vicente, J. L. Avina, L., Torres-Rodriguez, A., Hargis, B. and Tellez. G. 2007. Effect of a lactobacillus sppbased probiotics culture product on broiler chicks performance under commercial conditions. International Journal of Poultry Science, 6(3): 154-156.

Yaman, H., Ulukanli, Z., Elmali, M. and Unal, Y. The effect of a fermented probiotic, the kefir, on intestinal flora of poultry domesticated geese (ANSER ANSER) Revue. Méd. Vét. 2006; 157: 379-386.

Zhang, A. W., Lee, D. B., Lee, S. K., Lee, K. W., An, G. H., Song, K. B. and Lee, C. H. 2005. Effects of yeast (Saccharomyces cerevisiae) cell components on growth performance, meat quality, and ileal mucosa development of broiler chicks. Poultry Science Association, Inc.

Kabir, S. M. 2005. "The role of probiotics in the poultry industry International journal of molecular sciences." The Agriculturists 2005, 3, 24-29. 15.

Patidar, S. K. and Prajapati, J. B. 1999. Effect of feeding Lactobacilli on serum antibody titer and faecal microflora in chicks.

International Organization for Standards (ISO). 15 November 1995, Posting date. Susceptibility testing of infectious agents and evaluation of performance of antimicrobial susceptibility testing devices. 1. Reference method for testing the in vitro activity of antimicrobial agents against rapidly growing anaerobic bacteria involved in infectious diseases. ISO 20776-1. International Organization for Standards, Geneva, Switzerland. http://www.iso.org/iso/iso_catalogue/ catalogue_tc/catalogue_detail.htm?csnumber $=41630$. 